\title{
Pavement Crack Detection by Ridge Detection on Fractional Calculus and Dual-thresholds
}

\author{
Song Hongxun, Wang Weixing, Wang Fengping, Wu Linchun and Wang Zhiwei \\ Shaanxi Road Traffic Intelligent Detection and Equipment Engineering \\ Technology Research Center, Chang 'an University, Xi'an, China \\ School of Information Engineering, Chang'an University, Xi'an, China \\ songhongx@163.com
}

\begin{abstract}
In this paper, a new road surface crack detection algorithm is proposed; it is based on the ridge edge detection on fractional calculus and the dual-thresholds on a binary image. First, the multi-scale reduction of image data is used to shrink an original image to eliminate noise, which can not only smooth an image but also enhance cracks. Then, the main cracks are extracted by using the ridge edge detection on fractional calculus in a grey scale image. Subsequently, the resulted binary image is further processed by applying both short and long line thresholds to eliminate short curves and noise for getting rough crack segments. Finally the gaps in cracks are connected with a curve connection function which is an artificial intelligence routine. The experiments show that the algorithm for pavement crack images has the good performance of noise immunity, accurate positioning, and high accuracy. It can accurately locate and detect small and thin cracks that are difficult to identify by other traditional algorithms.
\end{abstract}

Keywords: Crack; Multiscale; Image enhancement; Ridge edge; Threshold

\section{Introduction}

In a time period, any road can be damaged gradually due to traffic loads and the other natural/artificial factors, and cracks are the most common road pavement surface defects that need to mend as early as possible. If the cracks are not handled properly, the damage will be more and more serious in the influence of repeated traffic pressure, rain and snow weathers [1]. The traditional pavement crack detection methods, based on artificial vision inspection, have the shortage of high labor work intensity, no uniform standard, slow speed, low accuracy and un-safety [2-3]. With the development of computers and digital image processing technology, pavement crack detection systems based on video images are widely used in the road maintenance. By the image technique, the road surface image acquisition is a procedure for the collecting data of the road condition, and then the pavement crack detection method will be used to evaluate road quality and to identify the pavement disease features [4-5]. These procedures can be used with the usage of intelligent transportation systems [6], and recently there have been many advanced image processing algorithms for crack detection, in which, the representative approaches are: a multiscale approach [7] was proposed to detect cracks based on Markov random fields for tracing cracks in road pavement surface images; in reference [8] authors suggested to adopt wavelet transform to detect cracks; Ayenu-Prah [9] studied a two-dimensional empirical mode decomposition crack detection method; the literature [10] presented the usage of Hough transform in crack detection, which can achieve better results because of Hough line transform making the good identification; Anders [11] developed a crack detection algorithm based on 
morphology; and others such as thresholding algorithms [12-13] were also applied for crack detection in a road surface. Some related research work, such as rock fracture detection and road detection, etc. were studied in [14-15].

Generally, it is similar to other application systems [16], a modern road crack detection system includes an optical imaging device such as a CCD camera on the rear of the trolley, and different image processing algorithms for detecting cracks in the video images. Due to the differences of the road surface materials and the degrees of smoothness, the system is often affected by the non-uniform illumination and system device itself. In a captured image, there exist a lot of noise and shadows. In addition, the crack objects are very thin and small by comparing to the large background in a pavement image. All these characteristics make the pavement crack detection and identification harder. So, this paper proposes a pavement crack detection algorithm based on the ridge edge detection and double thresholds for boundary connection. According to the characteristics of the crack images, the algorithm adopts multiscale technique to analyze the crack images by combining the different morphological operators, which can not only smooth images but also sharpen cracks. The main steps are: (1) the main cracks are extracted by a special ridge edge detection algorithm based on fractional calculus; (2) both small and large length thresholds are used for burr elimination and removal; (3) the gaps in cracks are identified and linked by the combination of different morphological operations; and (4) each of the crack targets is analyzed and refined on the basis of the reference images with different scales.

\section{Image Transform from Fine Scale to Course Scale}

As normal, the road image acquired through the system device contains two types of objects: the pavement background and cracks (target objects). By referring the literatures [17-18], the pavement image features can be summarized as follows:

(1) A crack is a set of lower grey value pixels respect to the pavement background. That is, the pixel gray value of the crack is much lower than that in its neighbors and the value is the local smallest.

(2) A crack normally has a linear characteristic in a certain direction, with the continuity in a space.

(3) Crack pixels, in the entire image, only occupy a very small proportion, about $0.1-1.0 \%$.

(4) Since the resolution of an image is up to $4000 \times 4000$ pixels in colors, therefore the small or thin cracks are easily disturbed by different road surface noise.

(5) Because the pavement material is rough, and the textures and 3-D geometries of the surface change a lot. These cause big speckle noise, low signal-to-noise ratio of cracks; low brightness contrast between the cracks and background, and poor crack pixel spatial continuity.

(6) Due to uneven illumination, cracks may show different gray values/colors in different locations in the pavement image.

Because of those image features, the pavement crack image enhancement is mainly for the expansion of the crack width and the reduction of image background noise. Since images are generally of the relatively large size, and an image includes a large amount of data, so the original image needs to shrink as a pretreatment. Due to the pavement material variation, the effects of a system, non-uniform illumination and the uneven brightness in an image, there is a lot of white noise in a pavement image. The cracks are only a small percentage proportional to the whole image, the algorithm for image shrinking by combining the morphological operations. The minimum neighborhood method is applied to shrink the image and extent the crack width. The concrete steps of the algorithm are as follows: 
1) Scale-down the length and width of an image $f 2 \mathrm{~m}$ times to get a new image fnew.

2) Position the shrunk image fnew $(i, j)$ corresponding pixels in the original image $f(i, j)$, and scan the neighboring pixels of $f_{0}(i, j), f_{1}(i, j), f_{2}(i, j) \cdots f_{n^{*} n-1}(i, j)$.

3) Calculate the mean value of the smallest $n$ neighboring pixel gray value, and assign the result value to the new image pixel fnew $(i, j)$, i.e.:

$$
f_{\text {new }}(i, j)=\frac{1}{n}\left[f_{0 \min }(i, j)+f_{1 \min }(i, j) \cdots+f_{(n-1) \min }(i, j)\right]
$$

From the results in Figure 1, it is obvious that the pretreatment can not only eliminate the white noise effectively but also strengthen the crack information. The different sized template can perform different results, and if the template size is too small, the result may be not so promising, but too large template used will blur the image and lower the brightness contrast which will affect the following detecting result. Therefore, $3 * 3$ template is selected for the experiments in this study.

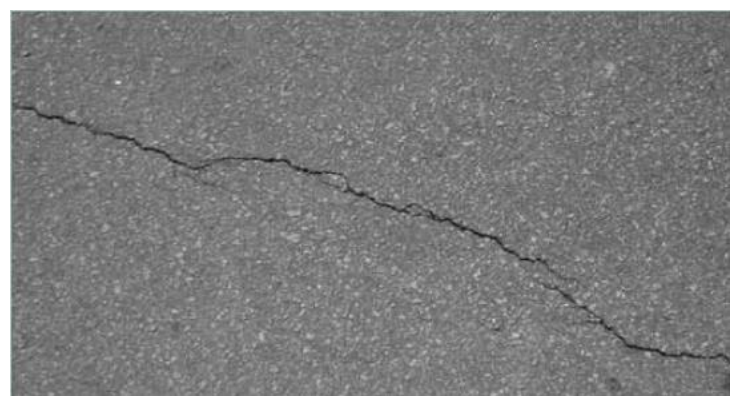

(a) Original image with white noise

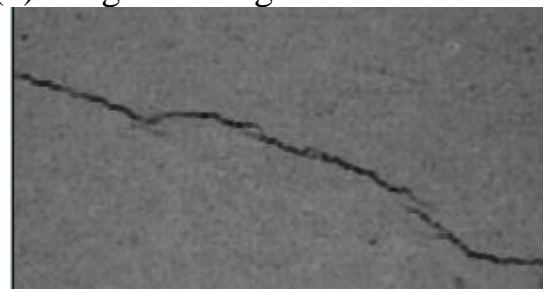

(c) Shrunk image by $3 \times 3$ Template

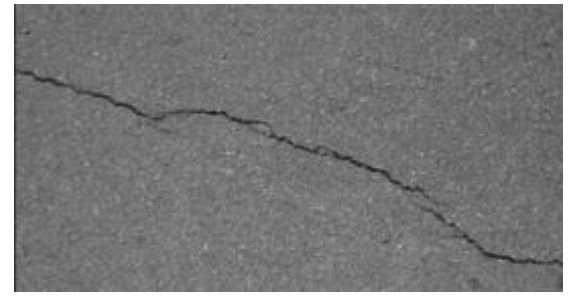

(b) Shrunk image by $2 \times 2$ Template

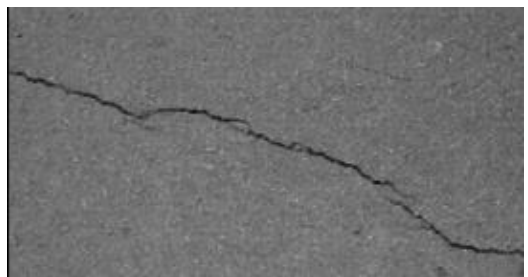

(d) Shrunk image by $5 \times 5$ Template

Figure 1. Original Pavement Crack Image and its Shrunk Images

\section{Ridge (Valley) Edge Detection based on Fractional Calculus}

\subsection{Fractional Calculus}

After image shrinking, the new image is smoother and the crack boundaries become weakened, and there exist some tiny black noise in the new image. As the fractional calculus can enhance weak boundaries and weaken the strong noise, the algorithm is applied in the ridge (valley) edge detection. The calculus (integral) effect to signal is shown in Figure 2:

As shown in Figure 2, the calculus algorithm can reduce the high-frequency signals and effectively enhance the low-frequency signals, which can be used to enhance the weak boundaries and eliminate some of the strong border particle noise signals in the image processing. So far, there is no unique definition of fractional calculus, scientists analyze and explore it from different aspects on the fractional calculus and get different definitions [19-20]. In all of those, Grümwald-Letnikov definition is widely used for digital image processing, and it is defined as: 


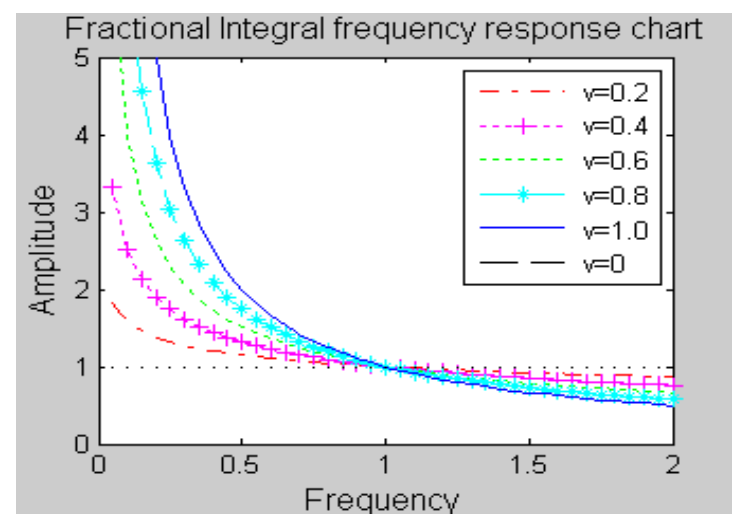

Figure 2. Amplitude-Frequency Graph of Fractional Integral

$$
{ }_{a}^{G} D_{t}^{v} f(t)=\lim _{h \otimes 0} h^{-v}{\underset{a}{r=0}}_{r}^{n}\left[{ }_{r}^{-v}\right] f(t-r h)
$$

Where, $f(t)$ is the signal, $\left[{ }_{r}^{-v}\right]=\frac{(-v)(-v+1) \cdots(-v+r-1)}{r !}$, the superscript G of ${ }_{a}^{G} D_{t}^{v}$ means the definition of Grümwald-Letnikov, $a$ and $t$ are the low and up boundary, and $v$ represents the order.

If $\mathrm{v}=-\mathrm{v}$ and $\mathrm{h}=1$, the definition of fractional integral can be released as:

$$
I^{v} f(t) ? f(t) \quad v f(t-1)+\frac{v(v+1)}{2} f(t-2)+\ldots+\frac{C(v+m)}{G(1+m) G(v)}
$$

$$
\Gamma(\alpha)=\int_{0}^{\infty} e^{-x} x^{\alpha-1} d x=(\alpha-1) \text { ! is a Gamma function. }
$$

\subsection{Ridge (Valley) Edge Detection Algorithm}

Because of the special features of pavement images, the results of traditional edge detection algorithms (such as Robert, Prewitt, Sobel, LOG, Canny etc.) are rough and cannot represent thin or small cracks accurately, and there exists many noise and spurious edges. Testing shows that it is difficultly to use the traditional edge detection algorithms for the pavement cracks, which is not conducive to the further extraction of cracks. Hence this paper proposes a ridge edge detection algorithm to locate small or thin cracks based on fractional calculus. Since the grey level of a pavement crack is lower than that in its surrounding neighborhood pixels. If a crack can be seen as a small "valley", the remaining question is how to locate and detect the "valley" by a special edge detection algorithm [21-22]. For which, the possible algorithm steps are as follows:

1) Scan an image $f(i, j)$ and construct $3 * 3$ sized templates in 8 directions, as shown in Figure 3 (a);
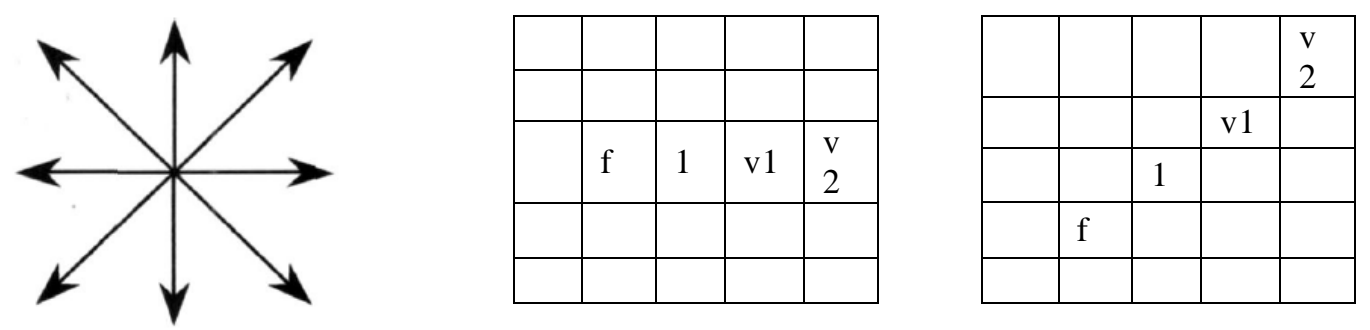
(a) 8 directions of algorithm

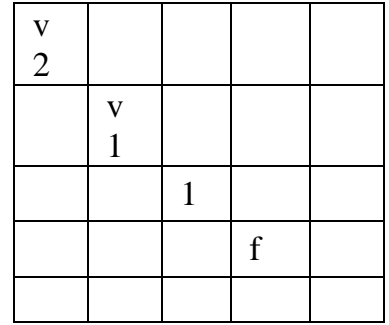

(d) $90^{\circ}$ direction

\begin{tabular}{|l|l|l|l|l|}
\hline & & & & \\
\hline & & & $\mathrm{f}$ & \\
\hline & & 1 & & \\
\hline & $\mathrm{v}$ & & & \\
& 1 & & & \\
\hline v2 & & & & \\
\hline
\end{tabular}

(g) $225^{\circ}$ direction (b) $0^{\circ}$ direction

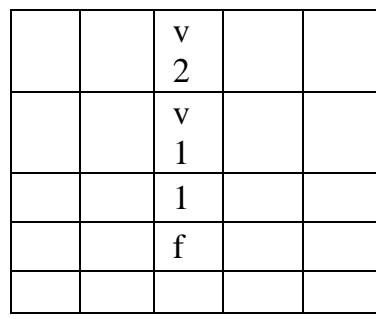

(e) $135^{\circ}$ direction

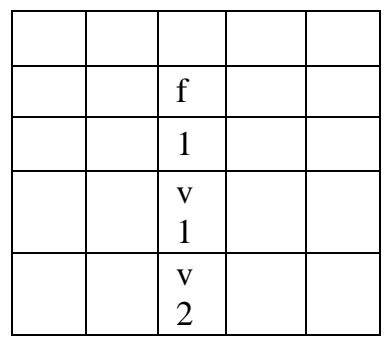

(h) $270^{\circ}$ direction (c) $45^{\circ}$ direction

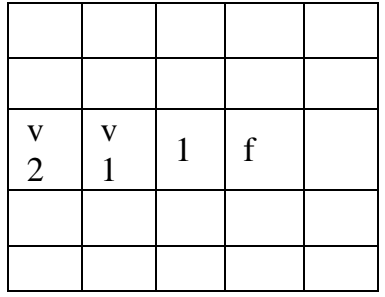

(f) $180^{\circ}$ direction

\begin{tabular}{|l|l|l|l|l|}
\hline & & & & \\
\hline & $\mathrm{f}$ & & & \\
\hline & & 1 & & \\
\hline & & & $\mathrm{v} 1$ & \\
\hline & & & & $\mathrm{v}$ \\
& & & & 2 \\
\hline
\end{tabular}

(i) $315^{\circ}$ direction

\section{Figure 3. Eight Templates based on Fractional Calculus in 8 Directions}

2) Calculate the 8 weighted mean values according to Figures $3(b)-(i) . v 1=v$, $\mathrm{v} 2=\mathrm{v}(\mathrm{v}+1) / 2, \mathrm{v}$ is the order of fractional integral. The mathematics expressions are presented in Eqs.(4)-(11):

$$
\begin{aligned}
& f_{0^{\circ}}(i, j)=\left[f(i+1, j)+f(i+2, j) * v+\left(f(i+3, j) * \frac{v(v+1)}{2}\right] /\left[1+v+\frac{v(v+1)}{2}\right]\right. \\
& f_{45^{\circ}}(i, j)=\left[f(i+1, j+1)+f(i+2, j+2) * v+\left(f(i+3, j+3) * \frac{v(v+1)}{2}\right] /\left[1+v+\frac{v(v+1)}{2}\right]\right. \\
& f_{90^{\circ}}(i, j)=\left[f(i, j-1)+f(i, j-2) * v+\left(f(i, j-3) * \frac{v(v+1)}{2}\right] /\left[1+v+\frac{v(v+1)}{2}\right]\right. \\
& f_{135^{\circ}}(i, j)=\left[f(i-1, j-1)+f(i-2, j-2) * v+\left(f(i-3, j-3) * \frac{v(v+1)}{2}\right] /\left[1+v+\frac{v(v+1)}{2}\right]\right. \\
& f_{180^{\circ}}(i, j)=\left[f(i-1, j)+f(i-2, j) * v+\left(f(i-3, j) * \frac{v(v+1)}{2}\right] /\left[1+v+\frac{v(v+1)}{2}\right]\right. \\
& f_{225^{\circ}}(i, j)=\left[f(i-1, j+1)+f(i-2, j+2) * v+\left(f(i-3, j+3) * \frac{v(v+1)}{2}\right] /\left[1+v+\frac{v(v+1)}{2}\right]\right. \\
& f_{270^{\circ}}(i, j)=\left[f(i, j+1)+f(i, j+2) * v+\left(f(i, j+3) * \frac{v(v+1)}{2}\right] /\left[1+v+\frac{v(v+1)}{2}\right]\right. \\
& f_{315^{\circ}}(i, j)=\left[f(i+1, j+1)+f(i+2, j+2) * v+\left(f(i+3, j+3) * \frac{v(v+1)}{2}\right] /\left[1+v+\frac{v(v+1)}{2}\right]\right.
\end{aligned}
$$

3) The 8 values are computed into 4 pairs: $0^{\circ}$ and $180^{\circ}, 45^{\circ}$ and $225^{\circ}, 90^{\circ}$ and $270^{\circ}$, $135^{\circ}$ and $315^{\circ}$, set a threshold value T, and identify the target pixel as "valley" in this direction if the two direction values of a pair are both higher than the threshold, i.e: $f_{m^{*} 45^{\circ}}(i, j)-f(i, j) \geq T \quad \& \& f_{m^{*} 45^{\circ}+180^{\circ}}(i, j)-f(i, j) \geq T$ then assign the 4 values as follow: 


$$
g_{m}(i, j)=\left\{\begin{array}{cc}
\frac{f_{m^{*} 45^{\circ}}(i, j)+f_{m^{*} 45^{\circ}+180^{\circ}}(i, j)}{2} & f_{m^{*} 45^{\circ}}(i, j)-f(i, j) \geq T \& \& \\
0 & f_{m^{*} 45^{\circ}+180^{\circ}}(i, j)-f(i, j) \geq T \\
\text { ot her s }
\end{array}\right.
$$

where, $m=0,1,2,3$.

4) Find out the maximum value of the 4 pair values, the pixel in the directions as the "valley" pixel;

5) Then, binarize the image according to the following equation:

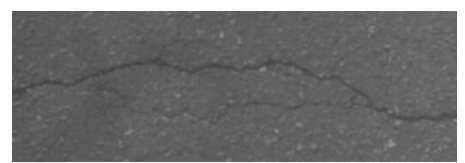

(a) Original image in

Figure 2 (c)

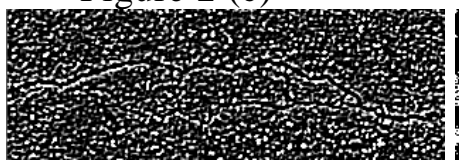

(d) Result of Sobel

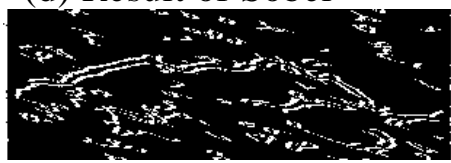

$(\mathrm{g})$

Canny $(\sigma / \mathrm{T} 1 / \mathrm{T} 2=.05 / 0$ $.4 / 0.9)$

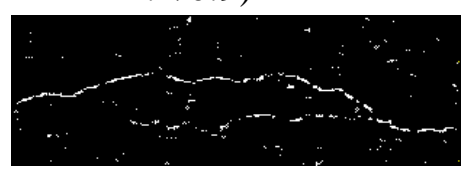

(j) New algorithm, 0.3 order

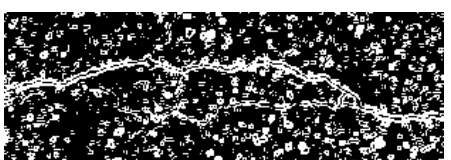

(b) Result of Robert

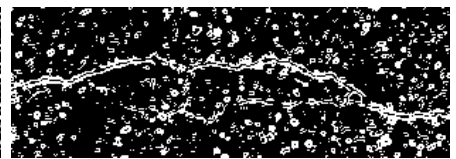

(e) Result of Laplace

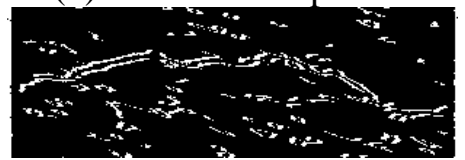

(h) Canny $(1 / 0.4 / 0.8)$

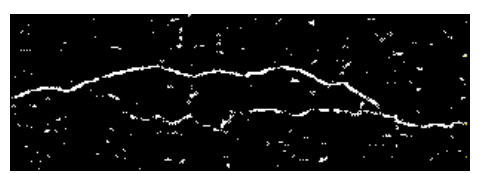

(k) 0.5 order

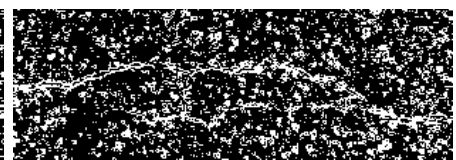

(c) Result of Prewitt

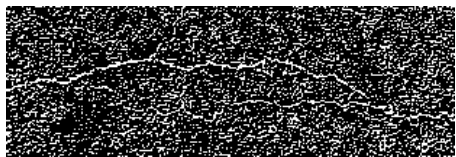

(f) Result of LOG

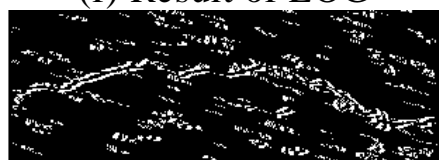

(i) Canny $(1 / 0.6 / 0.9)$

Figure 4. Comparison between Conventional Edge Detection Algorithms and New Algorithm

$f(i, j)=\left\{\begin{array}{cc}255 & g(i, j) \geq T^{\prime} \\ 0 & \text { else }\end{array}\right.$

To prove and validate the effect of the new algorithm, the other pavement images are selected testing, as one example, the comparison between the conventional edge detection algorithms and the new algorithm is shown in Figure 4.

\section{Short-term Noise Elimination and Dual-thresholds for Edge Connection}

Short-term, refers that its length is less than the tracking step $T$, and the disconnection is in a line in which the length is not less than $T$ but exists gaps [23]. After the valley edge detection above, there exist a lot of short-term noise in the binary image; and the determination whether it should be eliminated or not is often based on its length, this mainly depends on the size of the selected threshold. If the 
threshold value is too small many short-term burrs cannot be eliminated, and a large number of crack information will be missing if the selected threshold is too large. To solve this problem, a double threshold method is used. Firstly a short length threshold is chosen to eliminate short-term burrs, and then a long length threshold is selected to eliminate the other noise. The result processed by the short length threshold retains the most part of the crack information, and using the long length threshold can eliminate almost all of noise. So, the final result can be obtained by combining the two thresholds, after a number of morphological operations.

\subsection{Short-term Noise Cancellation}

The short-term noise can be roughly divided into three categories: Non-closed short-term curves, isolated pixel noise and Closed loop short-terms. A typical existing short-term elimination method is to track a line or a curve from one endpoint to another, which can deal with the above first and second types of noise under a certain condition, but cannot eliminate the third type of noise because for which there is no endpoint. For the third type of noise, the objects are thinned and labeled firstly. Then, the area and elongation of each labeled object are calculated. Subsequently, for the objects having the area less than $A$ or the elongation less than $E$, they are deleted. Where, $A$ and $E$ are determined by experience. As one example in Figure 5, the original binary includes three cracks with gaps, the black blocks and three types of noise are involved in the image; to delete the above noise, the thinning operation needs to operate as shown in Figure 5(b); a threshold 30 is used to delete the first two types of noise (see Figure 5(c)); for the third type of noise, the objects are labeled, then the area and elongation of each object are calculated, if the area is less than 50, or the elongation (length/width) is less than 3 , the object is removed, as shown in Figure 5(d), where the gaps on the cracks are linked automatically, based on the gap length and orientation.

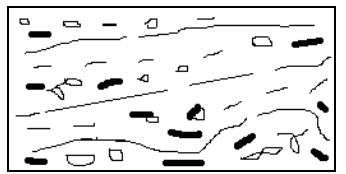

(a) Binary image

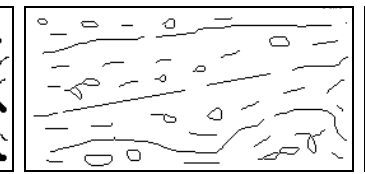

(b) Thinning result

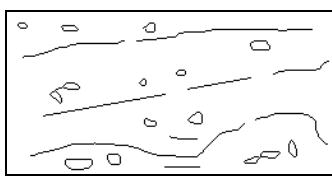

(c) Third type of noise

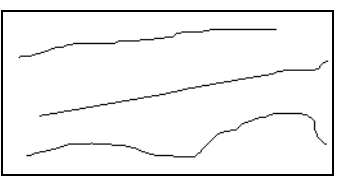

(d) Gap linking result

Figure 5. Removal of Three Types of Noise and Gap Linking in Cracks

\subsection{Dual-threshold Gap Linking}

There exist some gaps after eliminating short-terms, and the gaps need to link. The traditional ways are to search for possible candidate points (the endpoints), and decide if the two points should be connected or not is based on the distance between a couple of the candidate points and their orientation difference. However, these methods exist two obviously insufficient points: (1) this greatly depends on the predecided distance and angle thresholds, but no unified standard threshold value can be selected; and (2) the connection path completely relays on subjective requirements, and there may be wrong and drain connections. Therefore, this paper proposes a dual threshold gap linking method which makes object thinning and then uses both the short and long length thresholds to eliminate the short-terms for getting background and target images respectively. Then, it links the gaps in the target image based on the reference background image, one example is in Fig.5(d), and the specific steps are as follows: 
1) Get the result image (background image) which retains the crack information by only eliminating small noise with a short length threshold. The background image is used as a reference image for crack gap linking.

2) Further use a long length threshold to eliminate the most short-terms and noise to get the target image, where, the main segments of the cracks are remained because of the much bigger the threshold, the more noise and short fragments in the image can be eliminated even there exist some loss of the useful edge information. The target image is used for the initial point detection for gap linking.

3) Apply B-spline function to smooth all the lines or curves (pixel values are of $255)$ both in background image and the target image. The aim is to obtain the relative stable orientation for each endpoint or line that may be the candidate point or line for the gap linking.

4) Link the crack gaps in the target image with the reference background image as follows:

a) Scan the target image $\mathrm{f}$, and search for an endpoint $f(i, j)$ in the target image and set it as the detecting point.

b) Inspect the 8 neighboring pixels of $f(i, j)$ in the background image $\mathrm{b}$, if there exists non-zero value pixel $b(i 1, j 1)$ in the 8 neighboring pixels but its corresponding point in the target image $\mathrm{f}$ is zero, then the pixel $f(i 1, j 1)$ value in the target image will be assigned to 255 as the next new inspecting point in the target image.

c) Set $f(i 1, j 1)$ as a new detecting point, repeat step b) until there is no linking point in the background image, i.e. the crack gap linking of starting from endpoint $f(i$, $j$ ) is completed .Then go back to step a) to find out the next crack gap linking endpoint until the whole image is completely scanned.

\section{Experimental Results and Analysis}

The In the experiments, 300 pavement crack images are tested. According to experience, the main parameters are selected as: 0.8 as fractional calculus order, 4 as valley detection threshold, 6 as threshold for the binary image, and 2 and 20 as the short and long length thresholds. The test results are shown in Figure 6.

The other similar three pavement crack images are illustrated too, the comparing results between the new algorithm and the traditional Otus algorithm are shown in Figure 7.

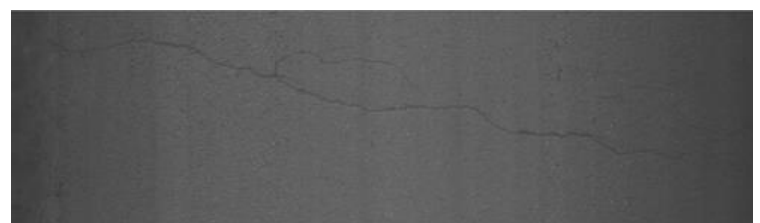

(a) Original pavement image

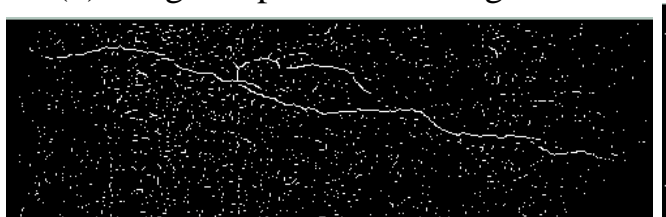

(c) Valley edge detection result

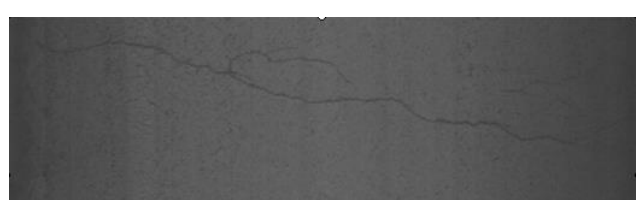

(b) $3 * 3$ template for Shrink image

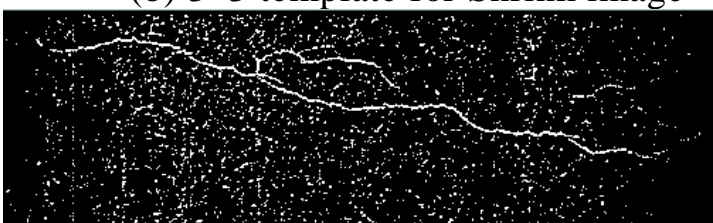

(d) Thinning result of (c) 


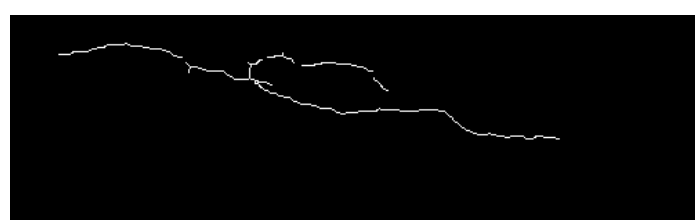

(e) Eliminating short-terms with threshold 2

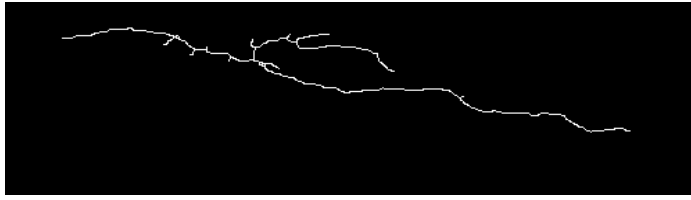

(g) Morphological processed result of (e)

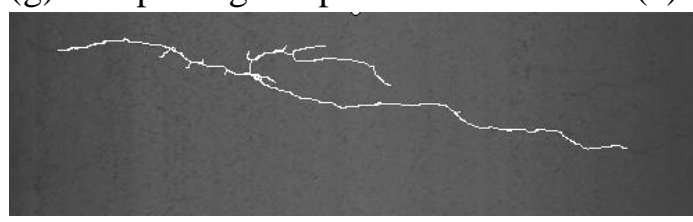

(i) Combination result of (b) and (h)

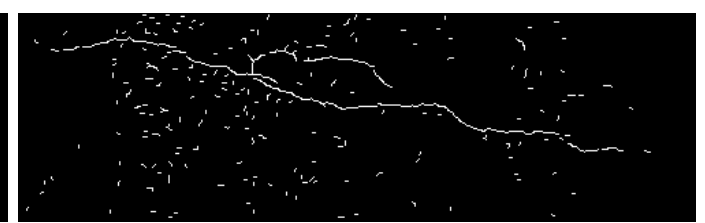

(f) Eliminating with threshold 20

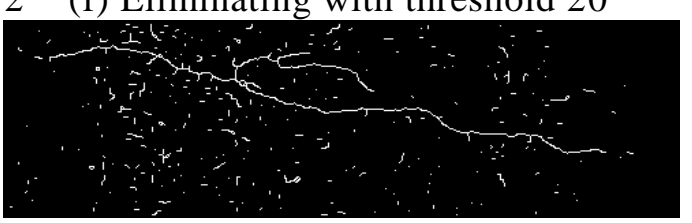

(h) Gap linking (Crack tracing result)

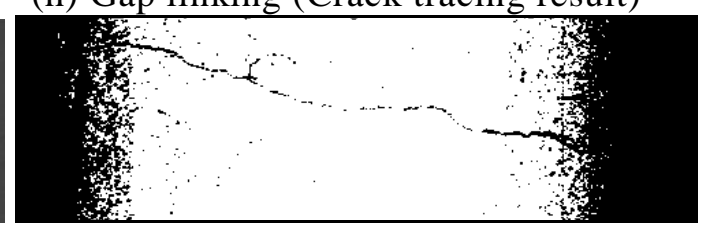

(j) Comparing result of Otus

Figure 6. Pavement Crack Tracing Procedure and Comparing to Others

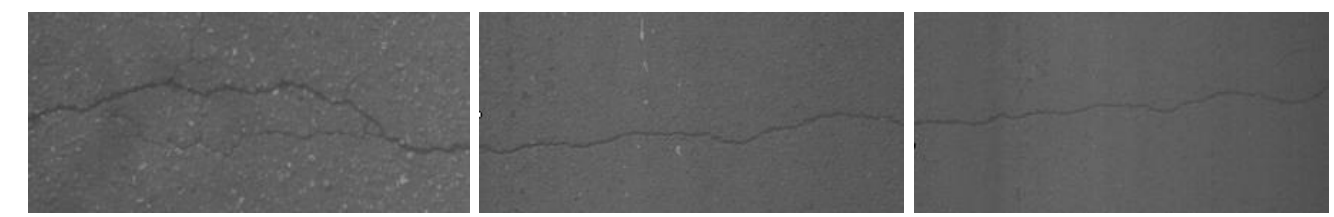

Three original crack images

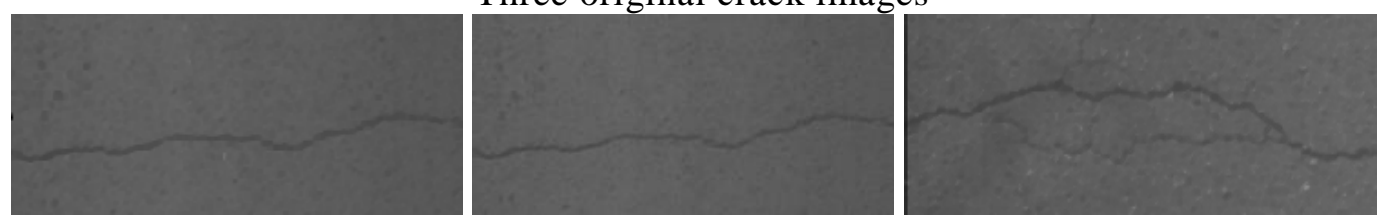

Shrunk images after reduced by 2 times
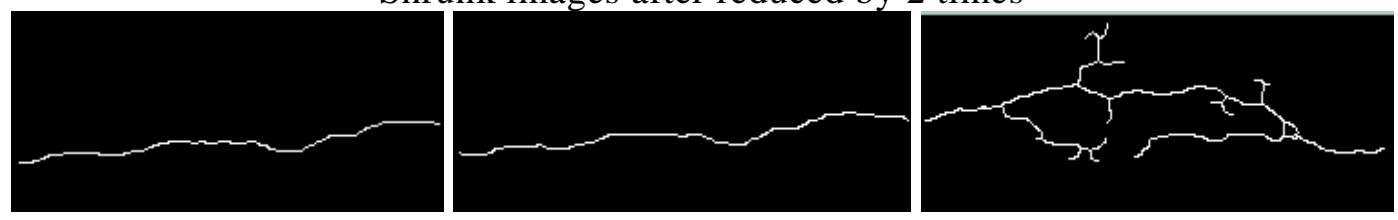

Detection results by new algorithm
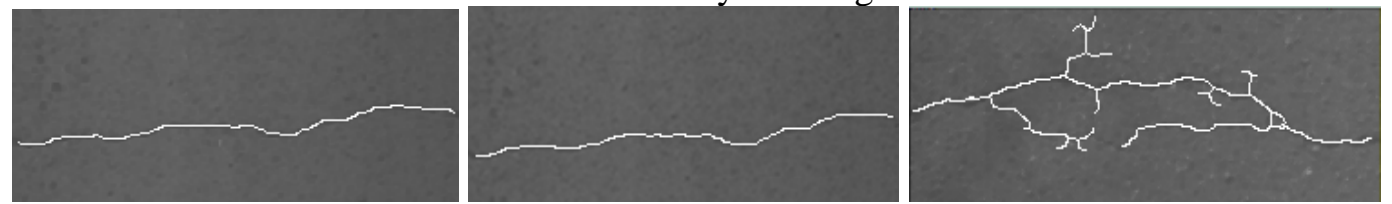

Positioning results of new algorithm

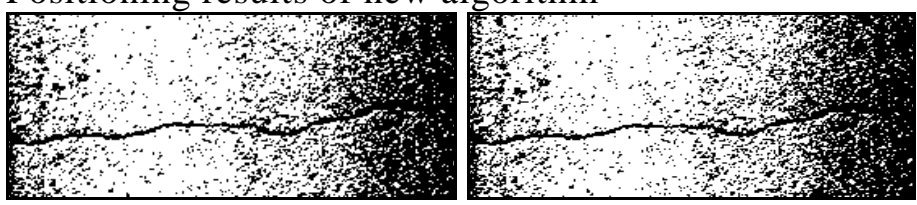

Detection results by Otus algorithm

$$
\text { Test I Test II }
$$

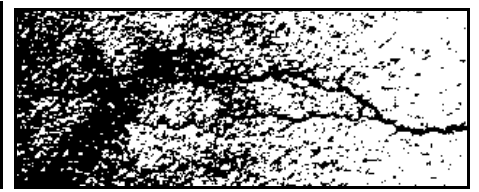

Figure 7. Three More Pavement Crack Image Processing Results

\section{Conclusions}


The pavement crack detection method based on valley edge detection of fractional calculus and dual-thresholds is proposed in this paper. The method designs a valley edge detection algorithm by using a group of fractional integral templates to detect small and thin cracks after the multi-scale image processing under the premise of no loss of crack information, then the thinning operation is performed on the objects in the binary image, subsequently, three types of noise are removed, the short length threshold is used to eliminate burr and get the background image, and the long length threshold is applied to get main crack segments as a target image. Finally it searches for the initial gap linking points in the target image, and links crack gaps by referring the information in the background image. Experimental results show that this method has good performance of anti-noise, high precision and accurate positioning, and it can meet the requirements of the small and tiny crack positioning and identification.

Since the test images are limited in this study, the further study should concentrate on color or grey level image segmentation by using new algorithms (e.g. graph based algorithms) [24], in addition, the crack shape analysis in a binary image should be important for determination if a detected object is a crack [25-26].

\section{Acknowledgements}

This research is financially supported by Shaanxi special funds for major scientific and technological innovation projects (No. 2007ZKC (-) 02-01), the National Natural Science Fund in China (grant no. 61170147), the Science and Technology Bureau of Shaanxi Province in China with number 2013KW03, and Special Fund for Basic Scientific Research of Central Colleges, Chang'an University in China (grant no. CHD2013G2241019) in China.

\section{References}

[1] Z. P. Ni, P. H. Tang and Y. Y. Xi, "A new method to pavement cracking detection based on the biological inspired model", 2012 International Conference on CSIP, (2012), pp. 755-758.

[2] K. C. P. Wang, W. G. Gong, X. Y. Li, R. P. Elliott and J. Daleiden, "Data analysis of real-time system for automated distress survey", Transportation Research Record: Journal of the Transportation Research Board, vol. 1806, (2002), pp. 101-109.

[3] Q. Q. Li, Q. Zou, D. Q. Zhang and Q. Z. Mao, "FoSA: F* seed-growing approach for crack-line detection from pavement images", Image and Vision Computing, vol. 29, (2011), pp. 861-872.

[4] H. Oliveira and P. L. Correia, "Automatic road crack detection and characterization", IEEE Transaction on Intelligent Transportation Systems, vol. 14, no. 1, (2013), pp. 155-168.

[5] S. Cafiso, A. Graziano, and S. Battiato, "Evaluation of pavement surface distress using digital image collection and analysis", Proc. 7th Int. Congr Adv. Civil Eng, (2006), Istanbul, Turkey.

[6] J. Zhang, F. Wang, K. Wang, W. Lin, X. Xu, and C. Chen, "Data-driven intelligent transportation systems: A survey”, IEEE Trans. Intell. Transp. Syst, vol. 12, no. 4, (2011), pp. 1624-1639.

[7] S. Chambon, P. Subirats, and J. Dumoulin, "Introduction of a wavelet transform based on 2D matched filter in a Markov random field for fine structure extraction: Application on road crack detection", Proc. IST/SPIE Electron Image. Sci. Technol, (2009), San Jose, CA, pp. 72510A-1-72510A-12.

[8] Y. Jeon, J. Yun, D. Choi, and S. Kim, " Defect detection algorithm for corner cracks in steel billet using discrete wavelet transform”, Proc. ICCAS-SICE, (2009), pp. 2769-2773.

[9] A. Prah, Albert, A. Okine and Nii, "Evaluating pavement cracks with bidimensional empirical mode decomposition", Eurasip J.Adv. Signal Process, vol. 1, (2008), pp. 861701-1-861701-7.

[10] S. Nashat, A. Abdullah, and M. Abdullah, "A robust crack detection method for non-uniform distributions of coloured and textured image”, Proc. IEEE Int. Conf. Image. Syst. Tech. (IST), (2011), pp. $98-103$.

[11] A. Landström and M. J. Thurley, "Morphology-based crack detection for steel slabs", IEEE Journal of Selected Topics In Signal Processing, vol. 6, (2012), pp. 866-875.

[12] Q. Li and X. Liu, "Novel approach to pavement image segmentation based on neighboring difference histogram method", Proc. IEEE CISP, (2008), Sanya, China, pp. 792-796.

[13] F. Liu, G. Xu, Y. Yang, X. Niu, and Y. Pan, "Novel approach to pavement cracking automatic detection based on segment extending", Proc. IEEE Int. Symp. Kam, (2008), Wuhan, China, pp. 610-614. 
[14] W. X. Wang, W S Li and X Yu, "Fractional differential algorithms for rock fracture images", The Imaging Science Journal, vol. 60, (2012), pp.103-111.

[15] W. X. Wang, Rock fracture image segmentation algorithms, Image Segmentation, Edited P. G. P. Ho, InTech Publisher (2011), pp. 459-488.

[16] W. X. Wang, "Colony image acquisition system and segmentation algorithms”, Optical Engineering, vol. 50, no. 12, (2011), pp. 123001

[17] Y. C. (James) Tsai, V. Kaul and A. Yezzi, "Automating the crack map detection process for machine operated crack sealer", Automation in Construction, vol. 31, (2013), pp. 10-18.

[18] F. M. Nejad and H. Zakeri, "A comparison of multi-resolution methods for detection and isolation of pavement distress", Expert Systems with Applications, vol. 38, (2011), pp. 2857-2872.

[19] P. I, "Fractional differential equations, mathematics in science and engineering", Academic Press, New York (1999), pp.150-158.

[20] K. B. Oldham and J. Spanier, "The fractional calculus", Academic Press, New York (1974).

[21] W. X. Wang, "Fragment Size Estimation without Image Segmentation", Imaging Science Journal, vol. 56, (2008), pp.91-96.

[22] W. Wang, F. Bergholm, and B. Yang, "Froth delineation based on image classification", Minerals Engineering, vol. 16, no. 11, (2003), pp.1183-1192.

[23] S. Yang, L. T. Shao, X. X. Guo, X. Liu and J. Zhang, "Skeleton and fractal law based image recognition algorithm for concrete crack", Chinese Journal of Scientific Instrument, vol. 33, no. 8, (2012), pp.18501854.

[24] W. X. Wang, X. Zhang, T. Cao, L. P. Tian, S. Liu and Z. W. Wang, "Fuzzy and Touching Cell Extraction on Modified Graph MST and Skeleton Distance Mapping Histogram", Journal of Medical Imaging and Health Informatics, vol. 4, no. 3, (2014), PP. (2156-7018/2014/4) 001-008, doi:10.1166/jmihi.2014.1264.

[25] W. X. Wang, W.G. Zhou and X. M. Zhao, "Airplane Extraction and Identification by Improved PCNN with Wavelet Transform and Modified Zernike Moments", The Imaging Science Journal, vol. 62, no. 1, (2014), pp.27-34.

[26] W. X. Wang, "Image analysis of particles by modified Ferret method- best-fit rectangle", Powder Technology, vol. 165, no. 1, (2006), pp. 1-10.

\section{Authors}

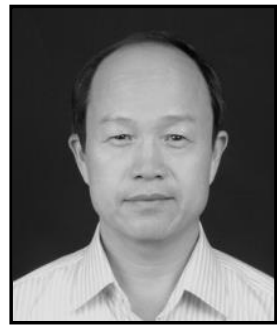

Song Hongxun, Associate professor in Chang'an University, China. In 1982, he obtained his Bachelor degree in Changchun University of Science and Technology, China, and in 1986, he obtained his Master degree in Xi'an institute of optics and precision mechanics of CAS, China. His interests include road traffic photoelectric detection technology, laser displacement sensors and photoelectric detection system, and image processing.

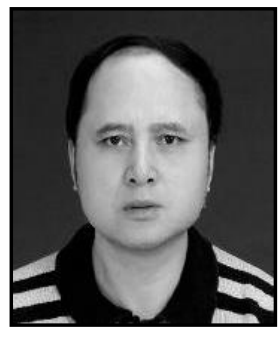

Wang Weixing, Professor in Information engineering. He obtained his $\mathrm{PhD}$ degree in 1997 at Royal Institute of Technology in Sweden, since 2001, he has been a PhD supervisor at Royal Institute of Technology, now he is a visiting professor at Chang'an University, and his interests involve Information engineering, Image processing and analysis, Pattern recognition and Computer Vision.

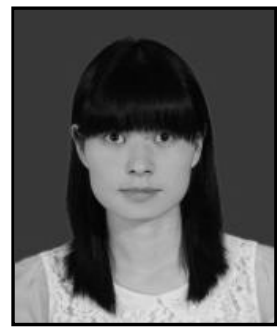

Wang Fengping, $\mathrm{PhD}$ student at School of Information Engineering, Chang'an University, China. In 2013, she obtained her Bachelor degree in Chang'an University. Her current interests are in the areas of feature extraction in remote sensing images, Image processing and analysis, and Computer vision. 
International Journal of Multimedia and Ubiquitous Engineering Vol.10, No.4 (2015) 